\title{
THE COMPLEXITY OF RIEMANN SURFACES AND THE HURWITZ EXISTENCE PROBLEM
}

\author{
ALDO-HILARIO CRUZ-COTA ${ }^{\bowtie}$ and TERESITA RAMIREZ-ROSAS
}

(Received 18 February 2012; accepted 6 May 2012)

\begin{abstract}
The complexity of a branched cover of a Riemann surface $M$ to the Riemann sphere $S^{2}$ is defined as its degree times the hyperbolic area of the complement of its branching set in $S^{2}$. The complexity of $M$ is defined as the infimum of the complexities of all branched covers of $M$ to $S^{2}$. We prove that if $M$ is a connected, closed, orientable Riemann surface of genus $g \geq 1$, then its complexity equals $2 \pi\left(m_{\min }+2 g-2\right)$, where $m_{\min }$ is the minimum total length of a branch datum realisable by a branched cover $p: M \rightarrow S^{2}$.
\end{abstract}

2010 Mathematics subject classification: primary 30F99; secondary 57M12.

Keywords and phrases: complexity of Riemann surfaces, branched covers between Riemann surfaces, Hurwitz existence problem.

\section{Introduction}

In this paper we study the simplest way in which a given Riemann surface can cover the Riemann sphere. The cover is allowed to have branching points and the simplicity of the branched cover is defined in terms of a complexity function.

Consider a branched cover of a Riemann surface $M$ to the Riemann sphere $S^{2}$. Let $B \subset S^{2}$ be the branching set of this cover, and let $X$ be the complement of $B$ in $S^{2}$. Then $X$ has a natural Riemann surface structure (as a domain of the Riemann sphere $S^{2}$ ). We are interested in studying the type of branched covers described above in which $X$ is a hyperbolic Riemann surface.

The complexity of a branched cover of a Riemann surface $M$ to the Riemann sphere $S^{2}$ is defined as the product of the degree of the cover and the hyperbolic area of the Riemann surface $X$. But, in order for this definition to make sense, we require that the Riemann surface $X$ be hyperbolic, or, equivalently, that the cardinality of $B$ be greater than or equal to three. (This will be assumed in the paper after Definition 4.3.)

We first learned of the previous definition of complexity of a branched cover in an unpublished paper ('The link volume', by Yo'av Rieck and Yasushi Yamashita), although these authors define it in the context of branched covers of 3-manifolds.

(C) 2012 Australian Mathematical Publishing Association Inc. 0004-9727/2012 \$16.00 
We define the complexity of a Riemann surface $M$ as the infimum of the complexities of all branched covers of $M$ to $S^{2}$. The main purpose of this paper is to study the following problem.

Problem 1.1. Find the complexity of a given Riemann surface $M$.

By the Gauss-Bonnet theorem, the complexity of a branched cover of $M$ to $S^{2}$ of degree $d \geq 1$ and with branching set of cardinality $n \geq 3$ is equal to $2 \pi d(n-2)$ (see Lemma 4.4). Therefore, the set of complexities of all branched covers of $M$ to $S^{2}$ is a subset of $\mathbb{Z} \pi$, the set of all integer multiples of $\pi$, which is a discrete subset of the real line. Thus, the complexity of $M$ is actually equal to the complexity of a branched cover of $M$ to $S^{2}$. In other words, Problem 1.1 is equivalent to the following.

Problem 1.2. Given a Riemann surface $M$, find a branched cover of $M$ to $S^{2}$ with minimal complexity.

Although the complexity of a branched cover is given by a simple formula, Problem 1.2 is difficult because, given a Riemann surface $M$, there are no known sufficient conditions for the existence of a branched cover of $M$ to $S^{2}$. Finding such conditions amounts to solving the (still open) Hurwitz problem, which we discuss below.

A branch datum is a 4-tuple $(M, n, d, \Pi)$ such that:

- $\quad M$ is a Riemann surface;

- $\quad n \geq 0$ and $d \geq 1$ are integers;

- $\quad \Pi$ is a collection of $n$ partitions of the integer $d$.

The total length of a branch datum $(M, n, d, \Pi)$ is defined as the sum of the lengths of the partitions in the collection $\Pi$.

A branched cover $p: M \rightarrow S^{2}$ naturally gives rise to a branch datum $(M, n, d, \Pi)$. Here, $n$ is the cardinality of the branching set $B \subset S^{2}, d$ is the degree of the cover, and $\Pi=\left(\Pi_{1}, \Pi_{2}, \ldots, \Pi_{n}\right)$ is the collection such that $\Pi_{i}$ is the partition of $d$ given by the degrees of the points on the preimage of the $i$ th branch point in $B$. A branch datum $(M, n, d, \Pi)$ is called realisable if it is associated to a branched cover $p: M \rightarrow S^{2}$.

Let $(M, n, d, \Pi)$ be a realisable branch datum, with $\Pi=\left(\Pi_{1}, \Pi_{2}, \ldots, \Pi_{n}\right)$. Define $m_{i}$ as the length of the partition $\Pi_{i}$ and let $m=\sum_{i=1}^{n} m_{i}$. By Theorem 3.2, the RiemannHurwitz formula becomes

$$
\chi(M)-m=d\left(\chi\left(S^{2}\right)-n\right) .
$$

A branch datum satisfying (1.1) is called compatible. Thus, every realisable branch datum is compatible, but the converse is not true (see [4, Corollary 6.4]). The classical Hurwitz problem asks which compatible branch data are actually realisable.

The Hurwitz problem stated above has a natural generalisation in which we consider branched covers of an arbitrary Riemann surface, not necessarily the Riemann sphere (see [11, Section 1] for precise statements). This more general problem was studied first by Hurwitz [7], and more recently by many authors, such as [1, 4, 6, 8, 10-12]. All instances of the general Hurwitz problem either have been solved or can be reduced 
to the version of the problem stated above, which is the only one that remains open (see [12, Section 2]).

A branched cover $p: M \rightarrow S^{2}$ of degree $d$ is called simple if the cardinality of the preimage $p^{-1}(y)$ is at least $d-1$ for all $y \in S^{2}$. We define the simple complexity of a Riemann surface $M$ as the infimum of the complexities of all simple branched covers of $M$ to $S^{2}$. We now state our two main results.

MaIn Theorem 1.3. Let $M$ be a connected, closed, orientable, hyperelliptic Riemann surface of genus $g>1$. Then the simple complexity of $M$ is equal to $8 \pi g$.

Main Theorem 1.3 gives an explicit formula for the simple complexity of a Riemann surface in terms of its genus. On the other hand, Main Theorem 1.4 below is not quite explicit: it gives a formula for the complexity of a Riemann surface, but this formula is in terms of an integer that is difficult to find. More precisely, we have the following result.

Main Theorem 1.4. Let $M$ be a connected, closed, orientable Riemann surface of genus $g \geq 1$. Let $m_{\min }$ be the minimum total length of a branch datum realisable by $a$ branched cover $p: M \rightarrow S^{2}$. Then the complexity of $M$ is equal to $2 \pi\left(m_{\min }+2 g-2\right)$.

Given a Riemann surface $M$, it is very difficult to find an explicit formula for the integer $m_{\min }$ from the statement of Main Theorem 1.4. The reason for this is that we do not know which branch data are realisable (by a branched cover of $M$ to $S^{2}$ ), which amounts to the fact that the Hurwitz problem is still open.

\section{Preliminaries}

Definition 2.1. A Riemann surface is a complex manifold of complex dimension one.

Throughout this paper, $M$ will denote a Riemann surface and $S^{2}$ will denote the Riemann sphere. All Riemann surfaces in this paper will be connected, closed and orientable. We denote the Euler characteristic of a Riemann surface $M$ by $\chi(M)$.

DeFinItion 2.2. A branched cover is a nonconstant holomorphic map between Riemann surfaces.

The following proposition is well known (see [3, Propositions 5 and 6 in Ch. 4]).

Proposition 2.3 [3]. Let $p: M \rightarrow N$ be a branched cover between Riemann surfaces.

(1) Then for each $x \in M$ there is a unique integer $k=k_{x} \geq 1$ such that we can find charts around $x$ in $M$ and $p(x)$ in $N$ in which the map $p$ is represented by the map $z \mapsto z^{k}$.

(2) Let $R$ be the set of all points $x$ in $M$ such that $k_{x}>1$. Then the set $R$ is finite.

(3) For each $y \in N$, the preimage $p^{-1}(y)$ is a finite subset of $M$.

Definition 2.4. We use the notation of Proposition 2.3.

- $\quad$ The set $R$ is called the ramification set of the branched cover $p$. A point in $R$ is called a ramification point of $p$. 
- $\quad$ The set $B=p(R)$ is called the branching set of the branched cover $p$. This set is always finite by Proposition 2.3(2). A point in $B$ is called a branch point of $p$.

- $\quad$ For each $x \in M$, the integer $k_{x} \geq 1$ is called the ramification index of $x$.

- The total ramification index of the branched cover $p$ is defined to be

$$
\mathcal{R}_{p}=\sum_{x \in M}\left(k_{x}-1\right)
$$

(This is a finite sum by Proposition 2.3(2).)

Given a branched cover $p: M \rightarrow N$, there is an integer $d \geq 1$ such that every point in $N$ has exactly $d$ preimages, if we count preimages with appropriate multiplicities. More precisely, we have the following lemma.

Lemma 2.5 [3]. Let $p: M \rightarrow N$ be a branched cover between Riemann surfaces. For each $y \in N$, we define the integer

$$
d(y)=\sum_{x \in p^{-1}(y)} k_{x} .
$$

Then the integer $d(y)$ does not depend on $y$. This integer will be called the degree of the branched cover $p$.

\section{The Riemann-Hurwitz formula and the Gauss-Bonnet theorem}

We now state the classical Riemann-Hurwitz formula.

Theorem 3.1 [9, Theorem 2.5.2]. Let $p: M \rightarrow N$ be a branched cover of degree $d$ between Riemann surfaces. Suppose that the genera of $M$ and $N$ are $g_{M}$ and $g_{N}$, respectively. Let $\mathcal{R}_{p}$ be the total ramification index of the branched cover $p$. Then

$$
2-2 g_{M}=d\left(2-2 g_{N}\right)-\mathcal{R}_{p} .
$$

We will also use the following alternative way of stating the Riemann-Hurwitz formula for branched covers of the Riemann sphere.

THEOREM 3.2. Let $M$ be a Riemann surface and let $p: M \rightarrow S^{2}$ be a branched cover of degree $d$. Suppose that branching set $B \subset S^{2}$ has cardinality $n$ and that the cardinality of the set $p^{-1}(B) \subset M$ is $m$. Then

$$
\chi(M)-m=d\left(\chi\left(S^{2}\right)-n\right) .
$$

Proof. Removing all the $n$ branch points from $S^{2}$ and all their $m$ preimages from $M$, we obtain that $p$ restricts to a genuine cover $p: M \backslash p^{-1}(B) \rightarrow S^{2} \backslash B$ of degree $d$. Therefore, $\chi\left(M \backslash p^{-1}(B)\right)=d\left(\chi\left(S^{2} \backslash B\right)\right)$, that is, $\chi(M)-m=d\left(\chi\left(S^{2}\right)-n\right)$.

We now define a special type of branched covers of the Riemann sphere.

Definition 3.3. A branched cover $p: M \rightarrow S^{2}$ of degree $d$ is simple if the cardinality of the preimage $p^{-1}(y)$ is at least $d-1$ for all $y \in S^{2}$. 
For simple branched covers of the Riemann sphere, the Riemann-Hurwitz formula simplifies to the following theorem.

Theorem 3.4. Let $p: M \rightarrow S^{2}$ be a simple branched cover of degree $d$ and with branching set of cardinality $n$. Let $g$ be the genus of $M$. Then

$$
2-2 g=2 d-n \text {. }
$$

Proof. By Theorem 3.1, it suffices to prove that $\mathcal{R}_{p}=n$. Combining the definition of a simple branched cover with Lemma 2.5, we obtain that:

- $\quad$ a point $x \in M$ is a ramification point of $p$ if and only if $k_{x}=2$;

- $\quad$ given a branch point $y \in S^{2}$, there exists a unique ramification point $x \in p^{-1}(y)$.

Thus, the cardinality of the ramification set $R$ equals the cardinality of the branching set $B$, which is $n$ by assumption. Therefore,

$$
\mathcal{R}_{p}=\sum_{x \in M}\left(k_{x}-1\right)=\sum_{x \in R}\left(k_{x}-1\right)=\sum_{x \in R}(2-1)=n .
$$

We recall the classical Gauss-Bonnet theorem.

Theorem 3.5 [2, Theorem V.2.7]. For M orientable with compact closure and smooth boundary,

$$
\int_{\partial M} \kappa_{g} d s+\int_{M} K d A=2 \pi \chi(M) .
$$

(Here $K$ is the Gaussian curvature of $M, \kappa_{g}$ is the geodesic curvature of the boundary $\partial M$ of $M$, and $\chi(M)$ is the Euler characteristic of $M$.

\section{4. $(d, n)$-branched covers of the Riemann sphere}

Definition 4.1. A branched cover $p: M \rightarrow S^{2}$ is said to be a $(d, n)$-branched cover (of the Riemann sphere) if the following properties are satisfied.

- $\quad$ The degree of $p$ is equal to $d$.

- $\quad$ The cardinality of the branching set of $p$ is equal to $n$.

Let $p: M \rightarrow S^{2}$ be a $(d, n)$-branched cover with branching set $B \subset S^{2}$. Then the surface $S^{2} \backslash B$ has a natural Riemann surface structure (induced by the Riemann surface structure of $S^{2}$ ). This Riemann surface is hyperbolic if and only if $n \geq 3$ (see [5, Theorem 27.12]).

Definition 4.3 below requires that the complement of the branching set in the Riemann sphere admit a hyperbolic structure. This will be assumed for the rest of the paper:

Notation 4.2. From now on, given a $(d, n)$-branched cover, we will always assume that $n \geq 3$.

We now define the complexity of $(d, n)$-branched covers. 
Definition 4.3. The complexity of a $(d, n)$-branched cover is defined as $d$ times the hyperbolic area of the complement of its branching set in $S^{2}$.

Using the Gauss-Bonnet theorem, we can easily find a formula for the complexity of a $(d, n)$-branched cover.

\section{LEMMA 4.4.}

(1) The hyperbolic area of the complement of $n \geq 3$ points in the Riemann sphere equals $2 \pi(n-2)$.

(2) The complexity of a $(d, n)$-branched cover equals $2 \pi d(n-2)$.

Proof. (1) Let $M_{n}$ be the complement of $n \geq 3$ points in the Riemann sphere, and let $A$ be its hyperbolic area. By the Gauss-Bonnet theorem (Theorem 3.5), $A=-2 \pi \chi\left(M_{n}\right)=$ $-2 \pi\left(\chi\left(S^{2}\right)-n\right)=2 \pi(n-2)$. (2) This follows from the definition of complexity.

\section{The main theorems}

We will need the following nonexistence result to prove one of our main theorems.

Lemma 5.1. There is no $(1, n)$-branched cover with $n \geq 3$.

Proof. Suppose that $p: M \rightarrow S^{2}$ is a $(1, n)$-branched cover with $n \geq 3$. Fix a point $y \in S^{2}$. By Lemma 2.5,

$$
\sum_{x \in p^{-1}(y)} k_{x}=1 .
$$

Since $k_{x} \geq 1$ for all $x \in M$, the last equality implies that the set $p^{-1}(y)$ consists of a unique point $x$ whose ramification index equals 1 . In other words, for each $y \in S^{2}$, the set $p^{-1}(y)$ contains no ramification points. This means that the branching set of $p$ is empty, contrary to our supposition that $p$ is a $(1, n)$-branched cover with $n \geq 3$.

Definition 5.2. A compact Riemann surface $M$ of genus $g>1$ is said to be hyperelliptic if there exists a double branched cover $p: M \rightarrow S^{2}$.

We now state one of our main theorems.

THEOREM 5.3. The simple complexity of a connected, closed, orientable, hyperelliptic Riemann surface of genus $g>1$ is equal to $8 \pi g$.

Proof. Let $M$ be a Riemann surface of genus $g>1$ and let $p: M \rightarrow S^{2}$ be a simple $(d, n)$-branched cover. By Theorem 3.4, $2-2 g=2 d-n$, so $n=2(d+g-1)$. Combining this with Lemma 4.4(2), we get that the complexity of $p: M \rightarrow S^{2}$ is equal to $2 \pi d(n-2)=4 \pi d(d+g-2)$.

For a fixed $g>1$, consider the function $f(d)=4 \pi d(d+g-2)$, defined for $d \geq 1$. Observe that, by definition, $f(d)$ is the complexity of a simple $(d, n)$-branched cover. The function $f(d)$ is increasing for $d \geq 1$ because $f^{\prime}(d)=4 \pi(d+g-2)+4 \pi d=$ $4 \pi(2 d+g-2) \geq 4 \pi$. (The last inequality holds because $d \geq 1$ and $g>1$.) 
Let $d_{\text {min }}$ be the minimal value of $d \geq 1$ such that there exists a simple $(d, n)$-branched cover $M \rightarrow S^{2}$ with $n \geq 3$. The previous paragraph shows that the simple complexity of $M$ is equal to $f\left(d_{\min }\right)$.

By Lemma 5.1, then there is no $(1, n)$-branched cover $M \rightarrow S^{2}$. This means that $d_{\min }>1$. On the other hand, since $M$ is hyperelliptic, then $d_{\min }=2$, and so $f\left(d_{\min }\right)=f(2)=8 \pi g$.

We would like to have a result similar to Theorem 5.3 for computing the complexity of a Riemann surface. We did not succeed in finding such a result, which is a difficult problem that is related to the Hurwitz problem (see Section 1). However, we have made some progress in this direction.

Theorem 5.4. Let $M$ be a connected, closed, orientable Riemann surface of genus $g \geq 1$. Let $m_{\min }$ be the minimum total length of a branch datum realisable by a branched cover $p: M \rightarrow S^{2}$. Then the complexity of $M$ is equal to $2 \pi\left(m_{\min }+2 g-2\right)$.

Proof. Let $p: M \rightarrow S^{2}$ be a $(d, n)$-branched cover and let $m$ be the total length of the branch datum associated to $p$. By Lemma 4.4, the complexity of $p$ is equal to $2 \pi d(n-2)$. On the other hand, by Theorem 3.2,

$$
\chi(M)-m=d\left(\chi\left(S^{2}\right)-n\right) .
$$

Therefore,

$$
m=\chi(M)-d(2-n)=2-2 g+d(n-2),
$$

and so

$$
m+2 g-2=d(n-2) .
$$

Hence, the complexity of $p$ is equal to $2 \pi(m+2 g-2)$. As the genus $g \geq 1$ is fixed, the minimal complexity of a branched cover $p: M \rightarrow S^{2}$ is equal to $2 \pi\left(m_{\min }+2 g-2\right)$. The conclusion now follows from the definition of complexity of a Riemann surface.

\section{Acknowledgements}

The authors would like to thank Daryl Cooper and Ilesanmi Adeboye for helpful discussions.

\section{References}

[1] K. Barański, 'On realizability of branched coverings of the sphere', Topology Appl. 116(3) (2001), 279-291.

[2] I. Chavel, Riemannian Geometry: A Modern Introduction, 2nd edn Cambridge Studies in Advanced Mathematics, 98 (Cambridge University Press, Cambridge, 2006).

[3] S. Donaldson, Riemann Surfaces, Oxford Graduate Texts in Mathematics, 22 (Oxford University Press, Oxford, 2011).

[4] A. L. Edmonds, R. S. Kulkarni and R. E. Stong, 'Realizability of branched coverings of surfaces', Trans. Amer. Math. Soc. 282(2) (1984), 773-790. 
[5] O. Forster, Lectures on Riemann Surfaces, Graduate Texts in Mathematics, 81 (Springer, New York, 1991), Translated from the 1977 German original by Bruce Gilligan, Reprint of the 1981 English translation.

[6] S. M. Gersten, 'On branched covers of the 2-sphere by the 2-sphere', Proc. Amer. Math. Soc. 101(4) (1987), 761-766.

[7] A. Hurwitz, 'Ueber Riemann'sche Flächen mit gegebenen Verzweigungspunkten', Math. Ann. 39(1) (1891), 1-60.

[8] D. H. Husemoller, 'Ramified coverings of Riemann surfaces', Duke Math. J. 29 (1962), 167-174.

[9] J. Jost, Compact Riemann surfaces, 3rd edn Universitext (Springer, Berlin, 2006).

[10] F. Pakovich, 'Solution of the Hurwitz problem for Laurent polynomials', J. Knot Theory Ramifications 18(2) (2009), 271-302.

[11] E. Pervova and C. Petronio, 'On the existence of branched coverings between surfaces with prescribed branch data. I', Algebr. Geom. Topol. 6 (2006), 1957-1985 (electronic).

[12] E. Pervova and C. Petronio, 'On the existence of branched coverings between surfaces with prescribed branch data. II', J. Knot Theory Ramifications 17(7) (2008), 787-816.

ALDO-HILARIO CRUZ-COTA, Department of Mathematics, Grand Valley State University, Allendale, MI 49401-9401, USA e-mail: aldo.h.cruz.cota@gmail.com

TERESITA RAMIREZ-ROSAS, Department of Mathematics, Grand Valley State University, Allendale, MI 49401-9401, USA e-mail: tere.ramirez.rosas@gmail.com 